\title{
Producción de Hidrógeno mediante digestión anaerobia de residuos de planta de jitomate
}

\section{Hydrogen production from anaerobic digestion of tomato's plant waste}

CAMARENA-MARTÍNEZ, Sarai†, MARTÍNEZ-MARTÍNEZ, Juan Humberto, SALDAÑA-ROBLES, Adriana y RUIZ-AGUILAR, Graciela M.L*

Universidad de Guanajuato, Carretera Irapuato-Silao Km. 9; C.P. 36500; Irapuato, Gto. México

ID $1^{\text {er }}$ Autor: Sarai, Camarena-Martínez / ORC ID: 0000-0002-0681-6364, CVU CONACYT ID: 495584

ID $1^{\text {er }}$ Coautor: Juan Humberto, Martínez-Martínez / ORC ID: 0000-0002-6325-3553, CVU CONACYT ID: 335602

ID $2^{\text {do }}$ Coautor: Adriana, Saldaña-Robles / ORC ID: 0000-0001-8871-1621, CVU CONACYT ID: 224323

ID $3^{\text {er }}$ Coautor: Graciela M.L, Ruiz-Aguilar / ORC ID: 0000-0001-9460-1429, CVU CONACYT ID: 122467

DOI: $10.35429 /$ JCPE.2019.19.6.1.12

Recibido: 20 de Abril, 2019; Aceptado 30 Junio, 2019

\section{Resumen}

Entre las alternativas de obtención de energía limpia que se están desarrollando, se reconoce el hidrógeno $\left(\mathrm{H}_{2}\right)$ como un portador de energía prometedor al poseer un alto rendimiento energético $(122 \mathrm{~kJ} / \mathrm{g})$ y se obtiene a partir de residuos lignocelulósicos mediante un proceso biológico. En el estado de Guanajuato se generan altas cantidades de residuos de planta derivados del cultivo de jitomate al ser el cultivo que mayormente se produce mediante agricultura protegida en el estado. Por lo que el objetivo del presente estudio fue el aprovechar residuos de planta de jitomate para la generación de hidrógeno a través del proceso de digestión anaerobia. Se evaluaron dos fuentes de inóculo, microflora nativa de la planta de jitomate y lodo anaerobio pretratado a $100^{\circ} \mathrm{C}$ por $24 \mathrm{~h}$; y cuatro medios minerales a un $\mathrm{pH}$ inicial de $6.5 \pm 0.2$ en experimentos en lote. El rendimiento más alto fue de 37.4 $\mathrm{mLH}_{2} / \mathrm{g}$ SV usando microflora nativa y medio mineral con extracto de levadura. Se logró una producción de hidrógeno similar a lo reportado en la literatura para otros residuos, destacando en nuestro caso, el ser innecesario el pretratamiento del sustrato o del inóculo. Por lo que la metodología propuesta resulta eficiente para la producción de hidrógeno a partir de la planta de jitomate.

Hidrógeno, Digestión anaerobia, Planta de jitomate

\begin{abstract}
Hydrogen $\left(\mathrm{H}_{2}\right)$ is recognized as a promising energy carrier among the alternatives for obtaining clean energy, since it has a high energy efficiency $(122 \mathrm{~kJ} / \mathrm{g})$ and can be obtained from lignocellulosic waste through a biological process. In the state of Guanajuato, high amounts of plant waste derived from tomato cultivation are generated because this is the crop mostly produced through protected agriculture. So, the objective of the present study was to consider tomato plant residues for the generation of hydrogen through the anaerobic digestion process. Two sources of inoculum, native microflora of the tomato plant and anaerobic sludge pretreated at $100^{\circ} \mathrm{C}$ for $24 \mathrm{~h}$; and four mineral media at an initial $\mathrm{pH}$ of $6.5 \pm 0.2$ in batch experiments, were evaluated. The highest yield was 37.4 $\mathrm{mLH}_{2}$ / g SV using native microflora and mineral media with yeast extract. Hydrogen production was found like those reported in the literature for other type of waste, highlighting the no-need to pretreat the substrate or inoculum. Therefore, the methodology propose is efficient to the hydrogen production from tomato plant residues.
\end{abstract}

Hydrogen, Anaerobic digestion, Tomato

Citación: CAMARENA-MARTÍNEZ, Sarai, MARTÍNEZ-MARTÍNEZ, Juan Humberto, SALDAÑA-ROBLES, Adriana y RUIZ-AGUILAR, Graciela M.L. Producción de Hidrógeno mediante digestión anaerobia de residuos de planta de jitomate. Revista de Energía Química y Física. 2019. 6-19: 1-12

\footnotetext{
* Correspondencia del Autor (Correo electrónico: gracielar@ugto.mx)

$\dagger$ Investigador contribuyendo como primer Autor.
} 


\section{Introducción}

El hidrógeno posee un alto contenido de energía por unidad de peso (122 MJ / Kg) y su combustión no genera emisiones de $\mathrm{CO}_{2}$, lo cual lo hace una alternativa de producción de energía amigable con el medio ambiente (Jain et al., 2010). Sin embargo, alrededor del $90 \%$ del hidrógeno producido se obtiene mediante procesos electroquímicos y termoquímicos que requieren altos consumos de energía y de combustibles fósiles (Kapdan y Kargi, 2006; Balat y Kirtay, 2010). Por ello, en los últimos años las investigaciones se han enfocado en la obtención de hidrógeno mediante procesos biológicos como la biofotólisis, la fotofermentación y la fermentación oscura ya que se pueden llevar a cabo en condiciones ambientales y requieren mucho menos consumo de energía (Guo et al., 2010).

La fermentación oscura es particularmente atractiva porque no necesita energía lumínica, no tiene problemas de inhibición por la presencia de oxígeno, requiere pequeños volúmenes de reactor y se puede llevar a cabo con un bajo costo de capital en comparación con los procesos de biofotolis y fotofermentación (Levin et al., 2004; Nath y Das, 2004; Pandey et al., 2013).Otra ventaja muy importante del proceso de fermentación oscura es que se pueden aprovechar residuos agrícolas como materia prima para la producción de hidrógeno.

Los residuos agrícolas son una fuente prometedora debido a su abundancia, distribución en todo el mundo y a su bajo costo (Guo et al., 2010). La utilización de bagazo de caña de azúcar, paja de trigo; planta de sorgo, paja de maíz, entre otros, se ha estudiado en los últimos años (Li y Chen., 2007; Ivanova et al., 2009; Fangkum y Reungsang, 2011; Pérez et al., 2015).

Existen otros cultivos que poseen un alto impacto económico y en la generación de residuos como es el cultivo de jitomate. México es el principal proveedor a nivel mundial de jitomate con una participación en el mercado internacional de $25.1 \%$ del valor de las exportaciones mundiales (SAGARPA, 2017). No obstante, desde su cultivo hasta su procesamiento se generan desechos que carecen de valor comercial y no tienen una adecuada disposición.
Hasta el momento, la información disponible sobre la producción de hidrógeno a partir de residuos de planta de jitomate se encuentra muy limitada y aun no se ha evaluado la variación de distintos factores que afectan el rendimiento de $\mathrm{H}_{2}$ a partir de este sustrato como el tipo de inóculo y la composición del medio. Estos son parámetros importantes que afectan la evolución de las vías fermentativas (Wang y Wan, 2009; De Gioannis et al., 2013). Bajo este panorama, se estudiaron dos fuentes de inóculo, microflora nativa de la planta de jitomate y lodo anaerobio pretratado a $100{ }^{\circ} \mathrm{C}$ por $24 \mathrm{~h}$; y cuatro medios minerales en experimentos en lote para conocer su efecto en la producción de hidrógeno.

\section{Metodología a desarrollar}

La metodología que se siguió se resume en la Figura 1. Se llevó a cabo la recolección y molienda del sustrato y para la evaluación de la producción de hidrógeno se consideraron dos variables: el tipo de inóculo y el tipo de medio mineral. El biogás producido se cuantificó mediante cromatografía de gases.

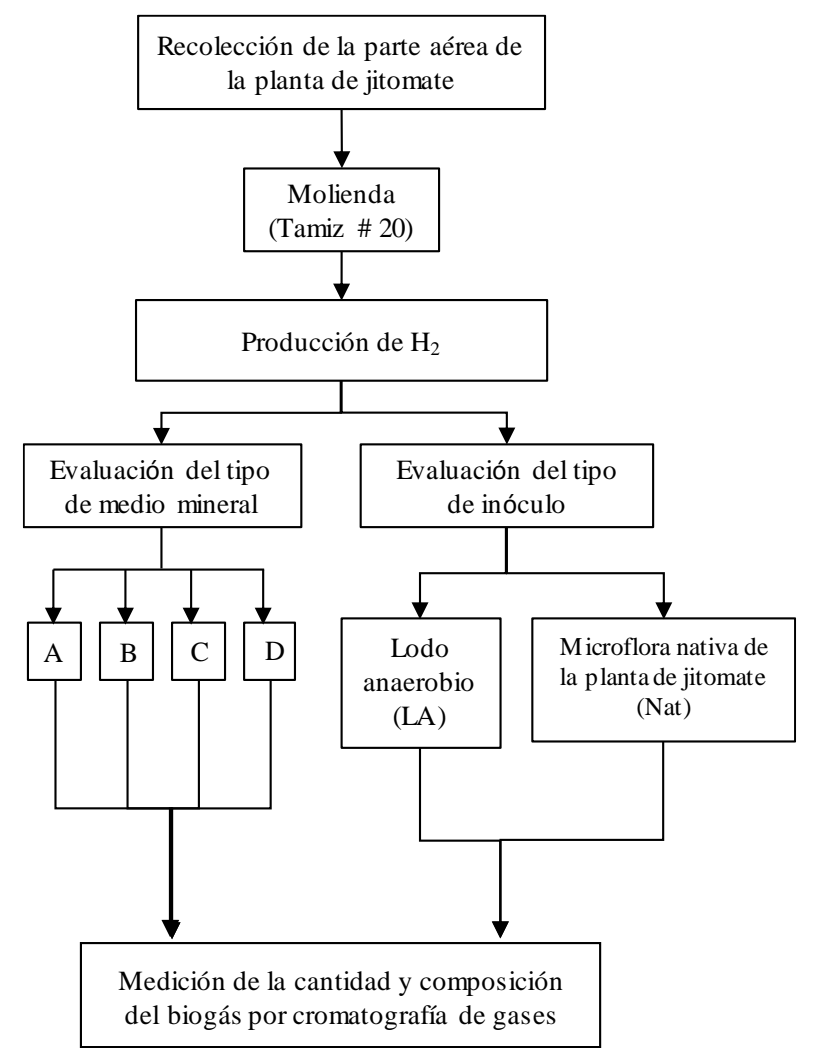

Figura 1 Metodología general para la producción de $\mathrm{H}_{2}$ a partir de planta de jitomate

Fuente: Elaboración Propia 


\section{Sustratos e inóculo}

El sustrato consistió en la parte aérea de la planta y fue recolectado al termino del ciclo de vida en las instalaciones de la Expo agroalimentaria ubicados en la ciudad de Irapuato, Gto. La variedad de planta de jitomate fue Saladette.

Las plantas se secaron bajo sol durante 15 días hasta llegar a una humedad entre 6 y $10 \%$. La planta seca se molió en un molino agrícola de martillos y se dejó almacenada a temperatura ambiente hasta su uso. Posteriormente para los análisis de caracterización y de producción de biogás se utilizó un molino marca SURTEK y un tamiz para conseguir un tamaño de partícula de aproximadamente $0.85 \mathrm{~mm}$.

El inóculo empleado consistió en lodo anaerobio que fue recolectado de un biodigestor tipo bolsa de geomembra de $1000 \mathrm{~L}$ el cual se alimenta con estiércol vacuno y es operado a temperatura ambiente. El biodigestor se encuentra ubicado en las instalaciones de la División Ciencias de la Vida. El inóculo recolectado se desgasificó a temperatura ambiente $\left(19.7 \pm 7.0^{\circ} \mathrm{C}\right)$ por un periodo de 10 días. Posteriormente este lodo se pretrato a 100 ${ }^{\circ} \mathrm{C}$ por un periodo de $24 \mathrm{~h}$ para emplearlo como inóculo LA en los experimentos de producción de hidrógeno.

\section{Métodos analíticos}

Para la caracterización del sustrato se realizaron los siguientes análisis: sólidos totales y sólidos volátiles, carbono orgánico, carbohidratos estructurales (celulosa, hemicelulosa y lignina), nitrógeno total Kjeldahl, demanda química de oxígeno y $\mathrm{pH}$. Además, se determinó el contenido de ST y SV del inóculo.

El contenido de carbono orgánico se realizó por el método de ignición de acuerdo con Dean, 1974, la determinación de carbohidratos estructurales se hizo mediante la metodología de van Soest et al., 1973, y el pH por medio del método reportado por Kang et al., 2014. El contenido de nitrógeno total Kjeldahl, DQO mediante el método de reflujo cerrado y contenido de sólidos totales y sólidos volátiles se realizó según los métodos estándares (APHA, 2005).

\section{Producción de $\mathrm{H}_{2}$}

Se evaluaron dos fuentes de inóculo, microflora nativa de la planta de jitomate (Inóculo Nat) y lodo anaerobio pretratado a $100{ }^{\circ} \mathrm{C}$ por $24 \mathrm{~h}$ (Inóculo LA); y cuatro medios minerales (A, B, C y D) cuya composición se indica en la Tabla 1. El medio mineral D corresponde al medio mineral reportado por Lara et al., 2014, y los medios minerales $\mathrm{A}, \mathrm{B}$ y $\mathrm{C}$ presentan una modificación con respecto al medio mineral $\mathrm{D}$, ya sea en la cantidad añadida de fosfatos y/o en la adición de extracto de levadura.

El medio mineral usualmente posee capacidad tampón (Angelidaki et al., 2004; Angelidaki et al., 2009) y esta puede estar dada por el buffer de fosfatos, el cual es el más empleado en los experimentos de producción de $\mathrm{H}_{2}$ por digestión anaerobia (Lin y Lay, 2004; Lin et al., 2011). Con la finalidad de aminorar costos y evitar una saturación de fosfatos en el medio (Cubillos et al., 2010; Zhao et al., 2010; Liu et al., 2015; Xu et al., 2016), se optó por utilizar la propuesta de Lara et al., $2014(0.075 \mathrm{M}$, medio mineral D) y otras dos concentraciones $0.02 \mathrm{M}$ (medio mineral B y C) y $0.1 \mathrm{M}$ (medio mineral A).

Por otro lado, se recomienda la adición de vitaminas al medio mineral, especialmente cuando se manejan residuos agrícolas para evitar inhibición por sustrato de la digestión (Angelidaki et al., 2004; Lindorfer et al. 2007; Angelidaki et al., 2009).

El extracto de levadura posee vitaminas del complejo B y además constituye una fuente de nitrógeno por lo que es muy usado en los medios minerales en conjunto con otras fuentes de nitrógeno (Fan et al., 2006; Ivanova et al., 2009; Li y Chen, 2007; Li et al., 2012). 


\begin{tabular}{|c|c|}
\hline Medio & Composición de medio mineral \\
\hline A & $\begin{array}{l}\mathrm{KH}_{2} \mathrm{PO}_{4} 4.6 \mathrm{~g}, \mathrm{~K}_{2} \mathrm{HPO}_{4} 10.0 \mathrm{~g}, \mathrm{NH}_{4} \mathrm{Cl} 6.0 \mathrm{~g} \text {, } \\
\mathrm{MgCl}_{2} \cdot 6 \mathrm{H}_{2} \mathrm{O} \quad 0.1 \mathrm{~g}, \quad \mathrm{CaCl}_{2} \quad 0.02 \quad \mathrm{~g} \text {, } \\
\mathrm{MnSO}_{4} \cdot 6 \mathrm{H}_{2} \mathrm{O} \quad 0.015 \mathrm{~g}, \mathrm{FeSO}_{4} \cdot 7 \mathrm{H}_{2} \mathrm{O} \quad 0.025 \mathrm{~g} \text {, } \\
\mathrm{CuSO}_{4} \cdot 5 \mathrm{H}_{2} \mathrm{O} 0.005 \mathrm{~g}, \mathrm{CoCl}_{2} \cdot 5 \mathrm{H}_{2} \mathrm{O} 0.125 \mathrm{mg} .\end{array}$ \\
\hline B & $\begin{array}{l}\mathrm{KH}_{2} \mathrm{PO}_{4} 2.0 \mathrm{~g}, \mathrm{~K}_{2} \mathrm{HPO}_{4} 0.92 \mathrm{~g}, \text { Extracto de } \\
\text { levadura } 1.5 \mathrm{~g} / \mathrm{L}, \mathrm{MgCl}_{2} \cdot 6 \mathrm{H} 2 \mathrm{O} 0.1 \mathrm{~g}, \mathrm{CaCl}_{2} \\
0.02 \mathrm{~g}, \mathrm{MnSO}_{4} \cdot 6 \mathrm{H}_{2} \mathrm{O} \quad 0.015 \mathrm{~g}, \mathrm{FeSO}_{4} \cdot 7 \mathrm{H}_{2} \mathrm{O} \\
0.025 \mathrm{~g}, \mathrm{CuSO}{ }_{4} \cdot 5 \mathrm{H}_{2} \mathrm{O} \quad 0.005 \mathrm{~g}, \mathrm{CoCl}_{2} \cdot 5 \mathrm{H}_{2} \mathrm{O} \\
0.125 \mathrm{mg} .\end{array}$ \\
\hline $\mathrm{C}$ & 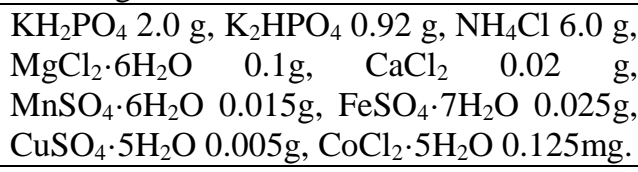 \\
\hline $\mathrm{D}$ & $\begin{array}{l}\mathrm{KH}_{2} \mathrm{PO}_{4} 4.8 \mathrm{~g}, \mathrm{~K}_{2} \mathrm{HPO}_{4} 6.98 \mathrm{~g}, \mathrm{NH}_{4} \mathrm{Cl} 6.0 \mathrm{~g}, \\
\mathrm{MgCl}_{2} \cdot 6 \mathrm{H}_{2} \mathrm{O} \quad 0.1 \mathrm{~g}, \quad \mathrm{CaCl}_{2} \quad 0.02 \quad \mathrm{~g}, \\
\mathrm{MnSO}_{4} \cdot 6 \mathrm{H}_{2} \mathrm{O} \quad 0.015 \mathrm{~g}, \mathrm{FeSO}_{4} \cdot 7 \mathrm{H}_{2} \mathrm{O} \quad 0.025 \mathrm{~g}, \\
\mathrm{CuSO}_{4} \cdot 5 \mathrm{H}_{2} \mathrm{O} \quad 0.005 \mathrm{~g}, \mathrm{CoCl}_{2} \cdot 5 \mathrm{H}_{2} \mathrm{O} 0.125 \mathrm{mg} .\end{array}$ \\
\hline
\end{tabular}

Tabla 1 Tipos de medio mineral evaluados para la producción de hidrógeno a partir de residuos de planta de jitomate

Fuente: Elaboración Propia

Los experimentos se llevaron a cabo en un régimen en lote a un $\mathrm{pH}$ inicial de $6.5 \pm 0.2$. Se adiciono $1.59 \pm 0.02 \mathrm{~g}$ de planta de jitomate seca y molida por cada $100 \mathrm{~mL}$ de mezcla (equivalente a $10.6 \mathrm{~g} \mathrm{SV}_{\text {sustrato/ }} / \mathrm{L}$ ). El volumen de trabajo fue de $80 \mathrm{~mL}$ manejando y un "espacio de cabeza" de $40 \mathrm{~mL}$. Los reactores se incubaron a $37^{\circ} \mathrm{C}$ durante un periodo de 15 días en condiciones estáticas. Para los tratamientos en los que se empleó Inóculo LA se añadió la cantidad $1.24 \pm 0.02 \mathrm{~g}$ de inóculo pretratatado y molido por cada $100 \mathrm{~mL}$ de mezcla (equivalente a $6.25 \mathrm{~g} \mathrm{SV}_{\text {inóculo }} / \mathrm{L}$ ). Cada tratamiento se realizó por duplicado.

El control negativo consistió en inóculo LA pretratado a $100{ }^{\circ} \mathrm{C}$ por $24 \mathrm{~h}$ sin añadir sustrato y se agregó el medio mineral Lara et al., 2014, para completar a un volumen de $80 \mathrm{~mL}$.

El análisis de cromatografía de gases para la detección de $\mathrm{H}_{2}, \mathrm{O}_{2}, \mathrm{~N}_{2}$, y $\mathrm{CH}_{4}$ se realizó tomando $30 \mu \mathrm{L}$ del gas presente en el "espacio de cabeza" de cada botella. Este se analizó en un cromatógrafo PerkinElmer ® modelo Clarus 580 , con una columna capilar Elite CG GSMOSIEVE $52(30 \mathrm{~m} \times 0.53 \mathrm{~mm} \times 50 \mu \mathrm{m})$ y un detector tipo TCD. Las temperaturas del inyector, horno y detector fueron 150, 50 y 200 ${ }^{\circ} \mathrm{C}$ respectivamente. Se utilizó argón como fase móvil a una presión de 14 psi.
Para analizar las diferencias significativas entre cada uno de los tratamientos se manejó un diseño factorial $2 \times 4$ y el método de Tukey con un valor alfa de $5 \%$ mediante el software de Statgraphics Centurion XVI.

\section{Resultados}

\section{Caracterización fisicoquímica}

Los resultados de la caracterización fisicoquímica de la parte área de la planta de jitomate se muestran en la Tabla 3. En cuanto a la determinación del contenido de ST y SV del lodo anaerobio fresco sin pretratar, se obtuvo $13.50 \pm 0.74 \%$ en base húmeda y $50.68 \pm 3.70 \%$ en base seca, respectivamente. Posteriormente este lodo se pretrató a $100{ }^{\circ} \mathrm{C}$ por un periodo de 24 h para emplearlo como inóculo LA en los tratamientos T1, T3, T5 y T7 (Tabla 2).

\section{Rendimiento de $\mathrm{H}_{2}$ y contenido de $\mathrm{H}_{2}$ en el biogas}

Solo en tres tratamientos se detectó producción de $\mathrm{H}_{2}$ en el biogás (T4, T7 y T8). En el tratamiento $\mathrm{T} 4$ se obtuvo el mayor rendimiento de $37.4 \mathrm{~mL} \mathrm{H}_{2} / \mathrm{g} \mathrm{SV}$ (Tabla 4).

La producción de $\mathrm{H}_{2}$ en el tratamiento T4 se detectó a partir de la medición realizada a las 63 horas. Para los tratamientos T7 y T8 se detectó hasta la medición realizada a las 135 horas. Esto indica que los microorganismos tardan más tiempo en adaptarse en las condiciones manejadas en los tratamientos $\mathrm{T} 7 \mathrm{y}$ T8 comparado con las establecidas en T4. En el caso de los tratamientos T4 y T7 no se observa producción de hidrógeno a las 327 h, en cambio en el tratamiento T8 la producción continua incluso después de haber transcurridos 15 días (Grafico 1).

En el tratamiento T4, durante las primeras 135 horas de incubación, se obtuvo una concentración de hidrógeno arriba del $60 \%$ y a partir de la medición realizada a las $183 \mathrm{~h}$ se observó una disminución significativa de la concentración. Para el tratamiento T7, el pico más alto de concentración se detectó a las $135 \mathrm{~h}$ y a partir de la siguiente medición se observó una disminución gradual de la concentración de hidrógeno (Grafico 2). 
Articulo

\begin{tabular}{|l|l|l|}
\hline Clave & Medio mineral & Inóculo \\
\hline T1 & A & LA \\
\hline T2 & A & Nat \\
\hline T3 & B & LA \\
\hline T4 & B & Nat \\
\hline T5 & C & LA \\
\hline T6 & C & Nat \\
\hline T7 & D & LA \\
\hline
\end{tabular}

Tabla 2 Tratamientos para evaluar la producción de $\mathrm{H}_{2}$ en experimentos en lote a partir de residuos de planta de jitomate

Fuente: Elaboración Propia

\begin{tabular}{|c|c|}
\hline Parámetros & Planta de jitomate \\
\hline $\mathrm{C} / \mathrm{N}$ & $19.86 \pm 0.67$ \\
\hline $\mathrm{ST}(\%)^{1}$ & $90.74 \pm 0.14$ \\
\hline $\mathrm{SV}(\%)^{2}$ & $73.59 \pm 0.14$ \\
\hline Celulosa $(\%)^{2}$ & $28.57 \pm 1.40$ \\
\hline Hemicelulosa $(\%)^{2}$ & $8.42 \pm 1.08$ \\
\hline Lignina $(\%)^{2}$ & $7.49 \pm 1.13$ \\
\hline Azúcares reductores ${ }^{2}$ & $1.70 \pm 0.18$ \\
\hline DQO ( mg/g SV) & $787.96 \pm 26.97$ \\
\hline $\mathrm{pH}$ & $6.60 \pm 0.06$ \\
\hline
\end{tabular}

Tabla 3 Caracterización fisicoquímica de la planta recolectada de jitomate

Fuente: Elaboración Propia

\begin{tabular}{|c|c|c|c|c|}
\hline Clave & $\begin{array}{c}\text { Medio } \\
\text { mineral }\end{array}$ & Inóculo & $\begin{array}{l}\text { Rendimiento } \\
\text { Biogás (mL /g } \\
\text { SV) }\end{array}$ & $\begin{array}{c}\text { Rendimiento } \\
\mathbf{H}_{2} \text { (mL /g } \\
\text { SV) }\end{array}$ \\
\hline $\mathrm{T} 1$ & A & LA & $25.5 \pm 4.2$ & 0.0 \\
\hline $\mathrm{T} 2$ & $\mathrm{~A}$ & Nat & $33.6 \pm 1.1$ & 0.0 \\
\hline T3 & $\mathrm{B}$ & LA & $74.9 \pm 1.5$ & 0.0 \\
\hline T4 & B & Nat & $57.8 \pm 4.8$ & $37.4 \pm 4.8$ \\
\hline T5 & $\mathrm{C}$ & LA & $27.6 \pm 3.0$ & 0.0 \\
\hline T6 & $\mathrm{C}$ & Nat & $6.0 \pm 0.4$ & 0.0 \\
\hline T7 & $\mathrm{D}$ & LA & $28.6 \pm 2.7$ & $5.0 \pm 2.0$ \\
\hline T8 & $\mathrm{D}$ & Nat & $25.2 \pm 7.1$ & $9.3 \pm 7.5$ \\
\hline
\end{tabular}

Tabla 4 Rendimientos de producción de biogás e hidrógeno obtenido en cada tratamiento para la producción de $\mathrm{H}_{2}$ en experimentos en lote a partir de residuos de planta de jitomate

Fuente: Elaboración Propia

En el caso del tratamiento $\mathrm{T} 8$, el contenido de $\mathrm{H}_{2}$ fue incrementando gradualmente durante el periodo de 15 días. El contenido de hidrógeno en el biogás total producido fue del $65 \%, 18 \%$ y $37 \%$ en los tratamientos T4, T7 y T8, respectivamente. De manera que en el tratamiento T4 se obtuvo la más alta concentración de hidrógeno en el biogás pero la producción más duradera se observó en el tratamiento T8 (Grafico 2). Alemahdi et al., 2015, evaluaron la producción de $\mathrm{H}_{2}$ usando como sustrato paja de arroz en codigestión con lodos activados de aguas residuales.
Ellos obtuvieron una concentración de hidrógeno en el biogás de $69.5 \%$, el cual es un valor cercano a lo obtenido en el tratamiento T4. Dhanasekar et al., 2018, reportaron un contenido de $\mathrm{H}_{2}$ en el biogás del $18 \%$ a partir de tallo de sorgo y usando la cepa Bacillus subtilis AUChE413 para llevar a cabo la fermentación. Este porcentaje es igual al obtenido en el tratamiento T7 de nuestro estudio. Otros autores mencionan rangos de concentración de $\mathrm{H}_{2}$ entre el 44 y $57 \%$ a partir de residuos de tallo de maíz (Zhang et al., 2007; Fan et al. 2008). Por lo cual nuestros resultados obtenidos son similares a lo reportado en la literatura, y en base a esto, el porcentaje de $\mathrm{H}_{2}$ en el biogás obtenido en el tratamiento $\mathrm{T} 4$ puede ser considerado de una alta concentración.

\section{Efecto del tipo de inóculo sobre la producción de hidrógeno}

El tipo de inóculo tuvo efecto significativo sobre la producción de hidrógeno $(\mathrm{P}<0.05)$, siendo el inóculo Nat el que mostró un mayor rendimiento (ver Anexo, Tabla 1 y Tabla 2).

Estos resultados difieren con lo reportado por Pérez, 2015, los cuales evaluaron cuatro consorcios microbianos como inóculo para la producción de hidrógeno a partir de paja de trigo. Los consorcios se obtuvieron a partir de muestras de suelo de jardín, rumen de vaca, lodo anaerobio y la microflora nativa de la paja de trigo. Se reportó que el más alto rendimiento se obtuvo de los reactores inoculados con lodo anaerobio seguido de la flora nativa de la paja de trigo. No obstante, en este estudio el lodo anaerobio no recibió ningún tipo de pretratamiento.

El menor rendimiento de producción de $\mathrm{H}_{2}$ a partir de lodos anaerobios (Inoculo LA) comparado con el obtenido a partir de la microflora nativa de la planta de jitomate (Inóculo Nat) pudo ser causado por el pretratamiento térmico aplicado al Inóculo LA que provoco una disminución en la diversidad microbiana. 


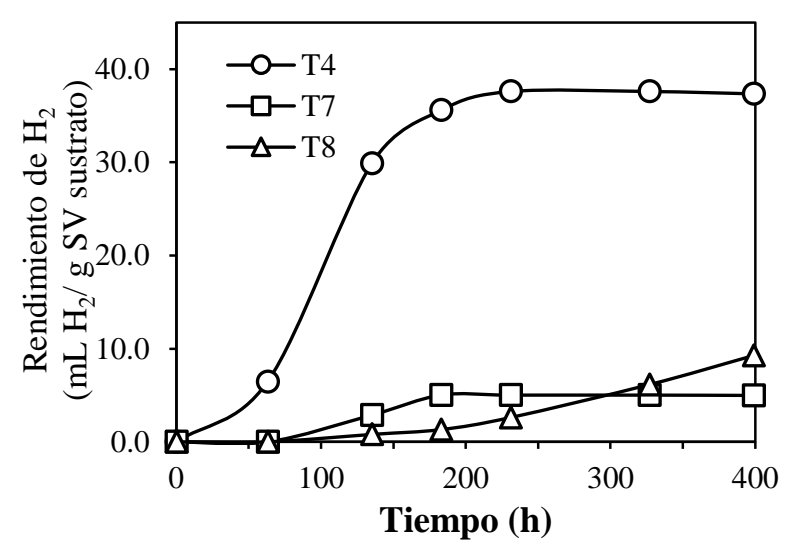

Grafico 1 Cinéticas de producción de hidrógeno acumulado en los tratamientos T4, T7 y T8 durante 15 días de incubación

Fuente: Elaboración Propia

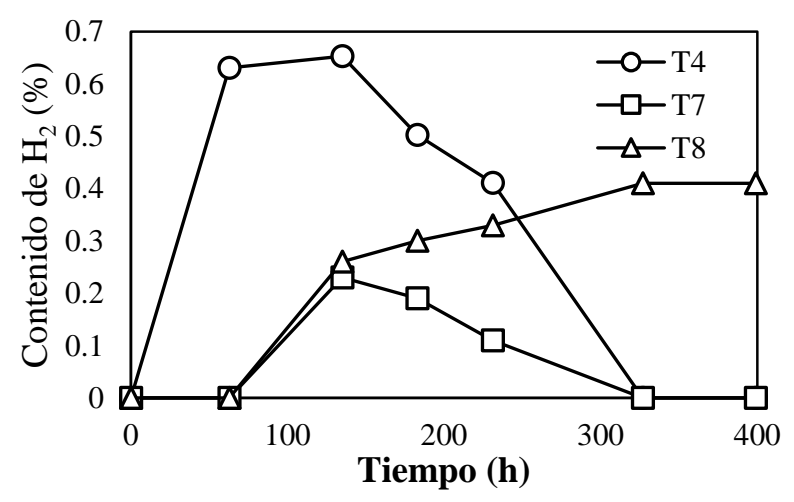

Grafico 2 Contenido de hidrógeno (\%) en los tratamientos T4, T7 y T8 durante 15 días de incubación Fuente: Elaboración Propia

En la literatura se mencionan diferentes condiciones de pretratamiento térmico con la finalidad de eliminar cepas consumidoras de hidrógeno las cuales no forman endoesporas y por ello no logran sobrevivir a altas temperaturas por tiempos prolongados (Fang et al., 2002; Liu et al., 2002; Duangmanee et al., 2007; Kanso et al., 2011).

Sin embargo, estos tratamientos térmicos pueden llegar a ser poco tolerantes para la mayor parte de los microorganismos presentes en los lodos anaerobios, lo que trae como consecuencia una disminución alta de la diversidad microbiana (Navarro et al., 2016). Esto implica que las vías metabólicas disponibles son menos diversas lo que dificulta la degradación del sustrato (Tracy et al., 2014; Vanwonterghem et al., 2014a). Asimismo, un consorcio productor de hidrógeno con menos especies podría disminuir la estabilidad ante perturbaciones o invasiones ambientales (Shade et al., 2012; Vanwonterghem et al., 2014b).
La aplicación de pretratamiento térmicos a más bajas temperaturas $\left(80-90{ }^{\circ} \mathrm{C}\right)$ puede ser recomendable para incrementar la concentración de cepas productoras de hidrógeno sin alterar significativamente las interacciones sintróficas entre cada grupo microbiano que conforma el inóculo.

\section{Efecto de la composición del medio mineral sobre la producción de hidrógeno}

De acuerdo con el ANOVA realizado, la composición del medio mineral tuvo un efecto significativo en el rendimiento de $\mathrm{H}_{2}(\mathrm{P}<0.05)$ (ver Anexo, Tabla 6 y Tabla 8).

No se detectó producción de hidrógeno al emplear el medio mineral A. Esto pudo deberse a la alta concentración de fosfatos que afecto la actividad metabólica de la microflora del Inóculo LA y del inóculo Nat, ya que este medio se preparó con la mayor concentración de fosfatos $(0.1 \mathrm{M})$. El fosfato es un nutriente inorgánico importante para el crecimiento microbiano. Las principales funciones del fosforo es la generación de energía en forma de trifosfato de adenosina (ATP) y la regulación de la capacidad amortiguadora del sistema celular (Lin y Lay, 2004).

Un aumento en la concentración de fosfato resulta en un aumento de la capacidad de las bacterias para producir hidrógeno. Sin embargo, concentraciones demasiado altas de fosfato podrían causar inhibición por el aumento de la producción de ácidos grasos volátiles, lo cual no es deseable ya que esto desvía los reductores celulares de la producción de hidrógeno (Lay et al., 2005; Bisaillon et al., 2006; Chandrasekhar et al., 2015). No obstante, en la medición de $\mathrm{pH}$ después de 15 días de incubación no se detectó una disminución significativa del mismo para estos tratamientos.

Según algunos protocolos establecidos para llevar a cabo pruebas de digestión anaerobia a escala laboratorio, se recomienda añadir una mezcla de vitaminas del complejo B (biotina, ácido fólico, riboflavina, ácido pantotenico, entre otros) en la preparación del medio mineral para evitar la inhibición del crecimiento de los microrganismos por la ausencia de estos nutrientes en sustratos no convencionales (Madigan et al., 2000; Angelidaki et al., 2004; Angelidaki et al., 2009). 
El extracto de levadura es un extracto soluble en agua formado por el autolisado de células de levaduras y es un producto rico en vitaminas especialmente del complejo B, aminoácidos y otros factores de crecimiento. En el tratamiento T4 se empleó inóculo Nat en medio mineral $\mathrm{B}$, el cual contiene extracto de levadura (1.5 g/L), y se observó una producción de hidrógeno más temprana con respecto al tratamiento T8, en el cual se usó inóculo Nat en medio mineral $\mathrm{D}$ que no contiene extracto de levadura. Esto indica que en nuestros experimentos el extracto de levadura juega un papel importante en la adaptación del inóculo Nat a las condiciones establecidas.

Por otro lado, el extracto de levadura constituye una fuente orgánica de nitrógeno al igual que la peptona. Li y Chen, 2007, evaluaron la producción de biohidrógeno a partir de paja de maíz mediante Clostridium butytiricum AS1.209 usando tres diferentes fuentes de nitrógeno, $\mathrm{NH}_{4} \mathrm{HCO}_{3}$, urea y extracto de levadura, en el medio mineral. En su estudio, los rendimientos de hidrógeno al usar fuentes de nitrógeno orgánico (urea y extracto de levadura) fueron mucho más altos que al emplear una fuente de nitrógeno inorgánico $\left(\mathrm{NH}_{4} \mathrm{HCO}_{3}\right)$. Esto también fue reportado por Ueno et al., 2001, y Ferchichi et al., 2005.

Kalil et al., 2009, reportaron que la adición de fuentes de nitrógeno orgánicas mejora el crecimiento celular con respecto a fuentes de nitrógeno inorgánico usando Clostridium acetobutylicum como inóculo y Zhang et al., 2014, mencionan que el incremento gradual de extracto de levadura disminuye el tiempo de la fase de latencia empleando lodo activado como inóculo.

Por lo que la adición de extracto de levadura promueve una mejor adaptación de los microorganismos para consumir el sustrato lo que conlleva a una mayor producción de hidrógeno o la obtención de biogás con una mayor concentración de hidrógeno (Kalil et al.2009; Alvarado-Cuevas et al., 2013; Zhang et al., 2014).

\begin{tabular}{|c|c|c|c|c|c|}
\hline $\begin{array}{c}\text { Sustra } \\
\text { to }\end{array}$ & $\begin{array}{l}\text { Pretratamie } \\
\text { nto de } \\
\text { sustrato }\end{array}$ & Inóculo & $\begin{array}{l}\text { Pretratamie } \\
\text { nto de } \\
\text { inóculo }\end{array}$ & $\begin{array}{c}\text { Rendimie } \\
\text { nto } \\
\text { (mL H } / 2 / g \\
\text { SV) }\end{array}$ & $\begin{array}{c}\text { Referenc } \\
\text { ia }\end{array}$ \\
\hline $\begin{array}{l}\text { Paja de } \\
\text { trigo }\end{array}$ & \begin{tabular}{|l|} 
Sin \\
pretratamien \\
to
\end{tabular} & $\begin{array}{l}\text { Lodo } \\
\text { anaerobio }\end{array}$ & $\begin{array}{l}\text { Sin } \\
\text { pretratamien } \\
\text { to }\end{array}$ & $10.6 \mathrm{~mL}$ & \multirow[t]{2}{*}{$\begin{array}{l}\text { Pérez et } \\
\text { al., } 2015\end{array}$} \\
\hline $\begin{array}{l}\text { Paja de } \\
\text { trigo }\end{array}$ & \begin{tabular}{|l|} 
Sin \\
pretratamien \\
to
\end{tabular} & \begin{tabular}{|l|} 
Microflora \\
nativa
\end{tabular} & \begin{tabular}{|l|} 
Sin \\
pretratamien \\
to
\end{tabular} & 7.0 & \\
\hline $\begin{array}{l}\text { Paja de } \\
\text { trigo }\end{array}$ & \begin{tabular}{|l|} 
Sin \\
pretratamien \\
to
\end{tabular} & \begin{tabular}{|l|} 
Composta \\
de estiércol \\
vacuno
\end{tabular} & Térmico & 1.0 & \multirow[t]{2}{*}{\begin{tabular}{|l|} 
Fan et \\
al., 2006
\end{tabular}} \\
\hline $\begin{array}{l}\text { Paja de } \\
\text { trigo }\end{array}$ & $\begin{array}{|lr|}\mathrm{HCl} & + \\
\text { Calentamien } \\
\text { to } & \text { de } \\
\text { microondas }\end{array}$ & \begin{tabular}{|l|} 
Composta \\
de estiércol \\
vacuno
\end{tabular} & \begin{tabular}{|l|} 
Térmico \\
\end{tabular} & 68.0 & \\
\hline $\begin{array}{l}\text { Paja de } \\
\text { trigo }\end{array}$ & \begin{tabular}{|l|} 
Enzimático / \\
epa \\
Trichoderm \\
$a$
\end{tabular} & $\begin{array}{l}\text { Lodo } \\
\text { anaerobio }\end{array}$ & $\begin{array}{|ll|}\text { Térmico } & \\
90^{\circ} \mathrm{C}, & 10 \\
\min & \\
\end{array}$ & 15.5 & $\begin{array}{l}\text { Quéméne } \\
\text { ur et al., } \\
2012\end{array}$ \\
\hline Pasto & \begin{tabular}{|l|} 
Ensilado l \\
fermentació \\
n con ácido \\
láctico pH \\
$<4.0$ \\
\end{tabular} & \begin{tabular}{|l|} 
Microflora \\
nativa
\end{tabular} & Ensilado & 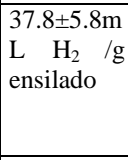 & $\begin{array}{l}\text { Li et al., } \\
2012\end{array}$ \\
\hline $\begin{array}{l}\text { Ensilad } \\
0 \quad \text { de } \\
\text { pasto }\end{array}$ & 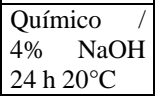 & \begin{tabular}{|l|} 
Lodo \\
anaerobio
\end{tabular} & \begin{tabular}{|l} 
Térmico, \\
hervido 30 \\
min
\end{tabular} & 6.5 & $\begin{array}{l}\text { Pakarine } \\
\text { n et al., } \\
2009\end{array}$ \\
\hline $\begin{array}{l}\text { Paja de } \\
\text { maíz }\end{array}$ & \begin{tabular}{|l|} 
Sin \\
pretratamien \\
to
\end{tabular} & \begin{tabular}{|l|}
$C$. \\
butyricum \\
AS1.209
\end{tabular} & \begin{tabular}{|l|} 
Sin \\
pretratamien \\
to
\end{tabular} & 9.0 & \multirow[t]{2}{*}{\begin{tabular}{|l} 
Li y \\
Chen., \\
2007
\end{tabular}} \\
\hline $\begin{array}{l}\text { Paja de } \\
\text { maíz }\end{array}$ & $\begin{array}{l}1.5 \mathrm{MPa} \\
\mathrm{min}\end{array}$ & \begin{tabular}{|l|}
$C$. \\
butyricum \\
AS1.209 \\
\end{tabular} & \begin{tabular}{|l|} 
Sin \\
pretratamien \\
to \\
\end{tabular} & 68.0 & \\
\hline $\begin{array}{l}\text { Planta } \\
\text { de } \\
\text { sorgo }\end{array}$ & $\begin{array}{l}130^{\circ} \mathrm{C} / 30 \\
\min \end{array}$ & \begin{tabular}{|l|} 
C. \\
saccharolyti \\
cus \\
(DSM8903) \\
\end{tabular} & $\begin{array}{l}\text { Sin } \\
\text { pretratamien } \\
\text { to }\end{array}$ & 32.4 & \begin{tabular}{|l} 
Ivanova \\
et al., \\
2009
\end{tabular} \\
\hline $\begin{array}{l}\text { Planta } \\
\text { de } \\
\text { jitomat } \\
\text { e } \\
\end{array}$ & \begin{tabular}{|l|} 
Sin \\
pretratamien \\
to
\end{tabular} & $\begin{array}{l}\text { Microflora } \\
\text { nativa }\end{array}$ & $\begin{array}{l}\text { Sin } \\
\text { pretratamien } \\
\text { to }\end{array}$ & \begin{tabular}{|l}
$37.4(\mathrm{~T} 4) \mathrm{y}$ \\
$9.3(\mathrm{~T} 8)$
\end{tabular} & \multirow[t]{2}{*}{$\begin{array}{l}\text { Este } \\
\text { estudio }\end{array}$} \\
\hline $\begin{array}{l}\text { Planta } \\
\text { de } \\
\text { jitomat } \\
\text { e } \\
\end{array}$ & $\begin{array}{l}\text { Sin } \\
\text { pretratamien } \\
\text { to }\end{array}$ & $\begin{array}{l}\text { Lodo } \\
\text { anaerobio }\end{array}$ & $\begin{array}{l}\text { Térmico } \\
100^{\circ} \mathrm{C}, 24 \mathrm{~h}\end{array}$ & $5.0(\mathrm{~T} 7)$ & \\
\hline
\end{tabular}

Tabla 5 Rendimiento de producción de hidrógeno a partir de residuos lignocelulosicos

Fuente: Elaboración Propia

En nuestro estudio se obtuvo un mayor rendimiento al emplear inóculo Nat en medio mineral B suplementado con extracto de levadura (T4) que al usar medio mineral C (T6) o medio mineral D (T8), tratamientos en los cuales se adicionó una fuente de nitrógeno inorgánica $\left(\mathrm{NH}_{4} \mathrm{Cl}\right)$.

No obstante, el inóculo LA mostró un comportamiento diferente con respecto al inóculo Nat, ya que éste no tuvo una mejora en la producción de hidrógeno al adicionar extracto de levadura. El único medio mineral con el cual se obtuvo producción de hidrógeno a partir de este consorcio bacteriano fue el medio mineral D. 


\section{Comparación de rendimientos de producción de hidrógeno con respecto a otros estudios}

El rendimiento obtenido en los tratamientos T4, T7 y T8 fue similar con respecto a lo reportado por otros autores los cuales indican un rendimiento de 1.0 a $68.0 \mathrm{~mL} / \mathrm{g} \mathrm{SV}$ a partir de residuos lignocelulósicos como paja de maíz, paja de trigo, planta de sorgo y ensilado de pasto (Li y Chen, 2007; Ivanova et al., 2009; Pakarinen et al., 2009; Li et al., 2012; Quéméneur et al., 2012; Pérez et al., 2015). Con respecto al estudio realizado por Pérez et al., 2015, en el que se usó como inóculo microflora nativa procedente del sustrato, nuestro rendimiento obtenido en el tratamiento $\mathrm{T} 4$ fue superior.

En cambio, en los estudios en los que se llevó a cabo un pretratamiento térmico o químico del sustrato con o sin pretratamiento del inóculo (Fan et al., 2006; Li y Chen, 2007), el rendimiento que se obtuvo en nuestro estudio a partir de planta de jitomate es inferior. Para los estudios en los cuales se llevó a cabo un pretratamiento biológico del sustrato, ya sea ensilado o enzimático ( $\mathrm{Li}$ et al., 2012; Quéméneur et al., 2012), el rendimiento conseguido en dichas investigaciones fue similar al nuestro (Tabla 4 y 5 ).

Es importante resaltar que el más alto rendimiento obtenido en nuestro estudio (37.4 $\mathrm{mL} \mathrm{H}_{2} / \mathrm{g} \mathrm{SV}$ ) se dio en el tratamiento en que se empleó microflora nativa (T4), es decir, no fue necesario añadir un inóculo externo. Además, no se llevó a cabo ningún tipo de pretratamiento térmico, biológico o químico del sustrato.

\begin{tabular}{|l|r|r|r|r|r|}
\multicolumn{1}{c}{ Fuente } & \multicolumn{1}{c}{$\begin{array}{c}\text { Suma de } \\
\text { Cuadrados }\end{array}$} & \multicolumn{1}{c|}{ Cuadrado } & Razón- Valor- \\
Medio & \multicolumn{1}{c|}{ F } & \multicolumn{1}{c|}{ P } \\
\hline Efectos Principales & & & & & \\
\hline A:Inóculo & 464.403 & 1 & 464.403 & 44.64 & $\mathbf{0 . 0 0 0 2}$ \\
\hline B:Medio Mineral & 956.527 & 3 & 318.842 & 30.65 & $\mathbf{0 . 0 0 0 1}$ \\
\hline Interacciones & & & & & \\
\hline Ab & 992.527 & 3 & 330.842 & 31.80 & $\mathbf{0 . 0 0 0 1}$ \\
\hline Residuos & 83.22 & 8 & 10.4025 & & \\
\hline Total (Corregido) & 2496.68 & 15 & & & \\
\hline
\end{tabular}

Tabla 6 Análisis de Varianza para evaluar el efecto del tipo de "Inóculo" y "Medio mineral" para la producción de $\mathrm{H}_{2}\left(\mathrm{~mL} \mathrm{H}_{2} / \mathrm{g} \mathrm{SV}\right)$ a partir de la digestión anaerobia de residuos de planta de jitomate

Fuente: Elaboración Propia

\section{Anexos}

Método: 95.0 porcentaje Tukey HSD

\begin{tabular}{|l|l|r|r|rr|}
\hline \multicolumn{2}{|c}{ Inóculo Casos } & \multicolumn{1}{c}{\begin{tabular}{c} 
Media \\
\multicolumn{1}{c}{ LS }
\end{tabular}} & \multicolumn{1}{c|}{$\begin{array}{c}\text { Sigma } \\
\text { LS }\end{array}$} & \multicolumn{2}{c|}{$\begin{array}{c}\text { Grupos } \\
\text { Homogéneos }\end{array}$} \\
\hline LA & 8 & 1.2 & 1.14031 & & X \\
\hline Nat & 8 & 11.975 & 1.14031 & & X \\
\hline
\end{tabular}

Contraste Sig. Diferencia +/- Límites
\begin{tabular}{|l|c|c|c|}
\hline LA - Nat & $*$ & $\mathbf{- 1 0 . 7 7 5}$ & 3.71878 \\
\hline
\end{tabular}
indica una diferencia significativa.

Tabla 7 Pruebas de Múltiple Rangos para la Producción de $\mathrm{H}_{2}\left(\mathrm{~mL} \mathrm{H} \mathrm{H}_{2} / \mathrm{g} \mathrm{SV}\right)$ por factor "Inóculo" a partir de la digestión anaerobia de residuos de planta de jitomate Fuente: Elaboración Propia

\section{Método: 95.0 porcentaje Tukey HSD}

\begin{tabular}{|l|l|r|r|r|}
\hline \multicolumn{1}{|c}{$\begin{array}{c}\text { Medio } \\
\text { mineral }\end{array}$} & Casos & \multicolumn{1}{c|}{$\begin{array}{c}\text { Media } \\
\text { LS }\end{array}$} & $\begin{array}{c}\text { Sigma } \\
\text { LS }\end{array}$ & \multicolumn{2}{c|}{$\begin{array}{c}\text { Grupos } \\
\text { Homogéneos }\end{array}$} \\
\hline C & 4 & 0 & 1.61265 & X \\
\hline A & 4 & 0 & 1.61265 & X \\
\hline D & 4 & 7.45 & 1.61265 & X \\
\hline B & 4 & 18.9 & 1.61265 & X \\
\hline
\end{tabular}

\begin{tabular}{|l|c|r|r|}
\multicolumn{2}{|c|}{ Contraste Sig. Diferencia +/- Límites } \\
\hline A - B & $*$ & $\mathbf{- 1 8 . 9}$ & 7.30076 \\
\hline A - C & & $\mathbf{0}$ & 7.30076 \\
\hline A - D & $*$ & $\mathbf{- 7 . 4 5}$ & 7.30076 \\
\hline B - C & $*$ & $\mathbf{1 8 . 9}$ & 7.30076 \\
\hline B - D & $*$ & $\mathbf{1 1 . 4 5}$ & 7.30076 \\
\hline C - D & $*$ & $\mathbf{- 7 . 4 5}$ & 7.30076 \\
\hline
\end{tabular}

$*$ indica una diferencia significativa.

Tabla 8 Pruebas de Múltiple Rangos para la Producción de $\mathrm{H}_{2}\left(\mathrm{~mL} \mathrm{H}_{2} / \mathrm{g} \mathrm{SV}\right)$ por factor "Medio mineral" a partir de la digestión anaerobia de residuos de planta de jitomate Fuente: Elaboración Propia

\section{Agradecimiento}

SCM agradece al Consejo Nacional de Ciencia y Tecnología (CONACYT) por su apoyo a través de la beca no. 442714 para realizar sus estudios de doctorado.

\section{Conclusiones}

El tipo de inóculo y la composición del medio mineral tuvieron efecto significativo sobre la producción de hidrógeno a partir de la fermentación anaerobia de residuos de planta jitomate. El inóculo procedente de la microflora nativa de la planta de jitomate (Inóculo Nat) presenta un mejor comportamiento en la producción de $\mathrm{H}_{2}$ con respecto al inóculo presente en el lodo anaerobio pretratado (Inóculo LA).

CAMARENA-MARTÍNEZ, Sarai, MARTÍNEZ-MARTÍNEZ, Juan Humberto, SALDAÑA-ROBLES, Adriana y RUIZ-AGUILAR, Graciela M.L. Producción de Hidrógeno mediante digestión anaerobia de residuos de planta de jitomate. Revista de Energía Química y Física. 2019. 
La adición de extracto de levadura mejoró la adaptación de los microorganismos que conforman el Inóculo Nat lo cual se vio reflejado en una mayor producción a un menor tiempo de incubación. Por otro lado, a una concentración de buffer fosfatos de $0.075 \mathrm{M}$ se observó producción de hidrógeno, pero al incrementar el valor a $0.1 \mathrm{M}$, se presentó una inhibición de la producción. Por tanto, el más alto rendimiento obtenido fue de $37.4 \mathrm{~mL} \mathrm{H}_{2} / \mathrm{g}$ $\mathrm{SV}$ al emplear Inóculo Nat y medio mineral con extracto de levadura a una concentración de buffer fosfatos de $0.02 \mathrm{M}$ (Medio mineral B). Para posteriores trabajos se recomienda realizar un proceso de optimización de la concentración de extracto de levadura y del buffer fosfatos en la producción de hidrógeno empleando microflora nativa de la planta de jitomate. En nuestro estudio no se llevó a cabo ningún pretratamiento térmico, biológico o químico al sustrato para lograr rendimientos similares o superiores a lo reportado en la literatura para otros tipos de residuos lignocelulósicos.

\section{Referencias}

Alemahdi, N., Che Man, H., Abd Rahman, N., Nasirian, N., \& Yang, Y. (2015). Enhanced mesophilic bio-hydrogen production of raw rice straw and activated sewage sludge by codigestion. International Journal of Hydrogen Energy, 40(46), 16033-16044.

Alvarado-Cuevas, Z. D., Acevedo, L. G. O., Salas, J. T. O., \& De León-Rodríguez, A. (2013). Nitrogen sources impact hydrogen production by Escherichia coli using cheese whey as substrate. New Biotechnology, 30(6), 585-590.

Angelidaki, I., Alves, M., Bolzonella, D., Borzacconi, L., Campos J.L., Guwya, J., Kalyuzhnyi,S., Jenicek, P., \& van Lie,r J.B. (2009). Defining the biomethane potential (BMP) of solid organic wastes and energy crops: a proposed protocol for batch assays. Water science and technology: a journal of the International Association on Water Pollution Research, 59(5), 927-934.

Angelidaki, I., \& Sanders, W. (2004). Assessment of the anaerobic biodegradability of macropollutants. Reviews in Environmental Science and Bio/Technology, 3(2), 117-129.
APHA, (2005). Standard methods for the examination of water and wastewater, 21st ed. APHA, Washington, DC.

Balat, H. \& Kirtay, E. (2010). Hydrogen from Biomass-Present Scenario and Future Prospects, Int. J. Hydrogen Energy, 35, 7416-7426.

Bisaillon, A., Turcot, J., \& Hallenbeck, P.C. (2006). The effect of nutrient limitation on hydrogen production by batch cultures of Escherichia coli. International Journal of Hydrogen Energy, 31, 1504-1508.

Chandrasekhar, K., Lee, Y.J., \& Lee, D.W. (2015). Biohydrogen Production: Strategies to Improve Process Efficiency through Microbial Routes. International Journal of Molecular Sciences, 16(12), 8266-8293.

Cubillos, G., Arrué, R., Jeison, D., Chamy, R., Tapia, E., \& Rodríguez, J., Ruiz-Filippi, G., (2010). Simultaneous effects of $\mathrm{pH}$ and substrate concentration on hydrogen production by acidogenic fermentation. Electronic Journal of Biotechnology, 13(1).

De Gioannis, G., Muntoni, A., Polettini, A., \& Pomi, R. (2013). A review of dark fermentative hydrogen production from biodegradable municipal waste fractions. Waste Management, 33 (6), 1345-1361.

Dean, W. (1974). Determination of Carbonate and Organic Matter in calcarcous sediments and sedimentary rocks by loss on ignition: Comparison with other methods. Journal of Sedimentary. Petrolology, 44 (1), 242-248.

Dhanasekar, R., \& Jonesh, S. (2018). Identification of a novel hydrogen producing bacteria from sugarcane bagasse waste. Biocatalysis and Agricultural Biotechnology, 15, 277-282.

Duangmanee, T., Padmasiri, S.I., Simmons J.J., Raskin L., \& Sung S. (2007). Hydrogen production by anaerobic microbial communities exposed to repeated heat treatment. Water Environ Res, 79, 975-983.

Fan, Y., Zhang, Y., Zhang, S., Hou, H., \& Ren, B. (2006) Efficient conversion of wheat straw wastes into biohydrogen gas by cow dung compost. Bioresour Technol, 97(3), 500-505. 
Fan, Y., Xing, Y., Ma, H., Pan, C., \& Hou, H. (2008). Enhanced cellulose-hydrogen production from corn stalk by lesser panda manure. International Journal of Hydrogen Energy, 33(21), 6058-6065.

Fang, H.H., Zhang, T., \& Liu, H. (2002). Microbial diversity of a mesophilic hydrogenproducing sludge. Appl Microbiol Biotechnol, $58,112-118$.

Fangkum, A. \& Reungsang, A. (2011). Biohydrogen production from sugarcane bagasse hydrolysate by elephant dung: effects of enitial $\mathrm{pH}$ and eubstrate eoncentration. International Journal of Hydrogen Energy, 36(14), 8687-8696.

Ferchichi, M., Crabbe, E., Gil, G.H., Hintz, W., \& Almadidy, A. (2005). Influence of initial $\mathrm{pH}$ on hydrogen production from cheese whey. Journal of Biotechnology, 120, 402-409.

Guo, X. M., Trably, E., Latrille, E., Carrère, H., \& Steyer, J.-P. (2010). Hydrogen production from agricultural waste by dark fermentation: A review. International Journal of Hydrogen Energy, 35(19), 10660-10673.

Ivanova, G., Rákhely, G., \& Kovács, K. L. (2009). Thermophilic biohydrogen production from energy plants by Caldicellulosiruptor saccharolyticus and comparison with related studies. Int J Hydrogen Energy 34 (9), 36593670

Jain, I.P., Lal, C., \& Jain,A. (2010). Hydrogen storage in $\mathrm{Mg}$ : a most promising material. Int $J$ Hydrogen Energy, 35, 5133-44.

Kalil, M. S., Alshiyab, H.S., Yusoff, W.M.W. (2008). Effect of nitrogen source and carbon to nitrogen ratio on hydrogen production using $\mathrm{C}$. acetobutylicum. American Journal of Biochemistry and Biotechnology, 4, 393-401.

Kang, J., Kim, K., Oh, G., \& Rhee, S. (2013). Analysis on biochemical methane potential of agricultural byproducts with different types of silage storage. Journal of Material Cycles and Waste Management, 16(3), 468-474.
Kanso, S., Dasri, K., Tingthong, S., \& Yuwadee, R. (2011). Diversity of cultivable hydrogenproducing bacteria isolated from agricultural soils, waste water sludge and cow dung. International journal of hydrogen energy, 36, 8735-8742.

Kapdan, I.K. \& Kargi, F. (2006). Bio-hydrogen production from waste materials. Enzyme Microbial Technology, 38, 569-582.

Lara, A.R., Sánchez, A., \& Valdez, I. (2014) Hydration treatments increase the biodegradability of native wheat straw for hydrogen production by a microbial consortium. International Journal of Hydrogen Energy, 39, 19899-19904.

Lay, J.J., Fan, K.S., Hwang, J.I., Chang, J.I., \& Hsu, P.C. (2005). Factors affecting hydrogen production from food wastes by Clostridiumrich composts. Journal of Environmental Engineering, 131, 595-602.

Levin, D.B., Pitt, L., \& Love, M. (2004). Biohydrogen production: prospects and limitations to practical application. Int $J$ Hydrogen Energy, 29, 173-185.

Li, D. \& Chen, H. (2007). Biological hydrogen production from steam exploded straw by simultaneous saccharification and fermentation. International Journal of Hydrogen Energy, 32 (12), 1742-1748.

Li, Y.C., Nissilä, M.E., Wu, S.Y., Lin, C.Y., \& Puhakka, J.A. (2012). Silage as source of bacteria and electrons for dark fermentative hydrogen production. International Journal of Hydrogen Energy, 37, 15518-15524.

Lin,, C.Y., \& Lay, C.H. (2004). Effects of carbonate and phosphate concentrations on hydrogen production using anaerobic sewage sludge microflora. International Journal of Hydrogen Energy, 29275-281.

Lin, P.J. Chang, J.S., Yang, L.H., Lin, C.Y., Wu, S.Y., Lee, K.S. (2011). Enhancing the performance of pilot-scale fermentative hydrogen production by proper combinations of HRT and substrate concentration. Int J Hydrog Energy 2011; 36: 14289-94. 
Lindorfer, H., Pérez López, C., Resch, C., Braun, R. \& Kirchmayr, R. (2007). The impact of increasing energy crop addition on process performance and residual methane potential in anaerobic digestion. Water Sci. Technol. 56 (10), $55-63$.

Liu, W.T., Chan, O.C., \& Fang, H.H.P. (2002). Microbial community dynamics during start-up of acidogenic anaerobic reactors. Water Resour Res, 36, 3203-3210.

Liu, B.-F., Xie, G.-J., Wang, R.-Q., Xing, D.-F., Ding, J., Zhou, X., Ren, N.-Q. (2015). Simultaneous hydrogen and ethanol production from cascade utilization of mono-substrate in integrated dark and photo-fermentative reactor. Biotechnology for Biofuels, 8(1), 8.

Madigan, M.T., Marinko, J.M., \& Parker, J. (2000). Brock Biology of Microorganisms. 9th edn. Prentice Hall, NY.

Nath, K. \& Das, D. (2004). Improvement of fermentative hydrogen production: various approaches. Appl Microbiol Biotechnol, 65, 520-529.

Navarro-Díaz, M., Valdez-Vazquez, I., \& Escalante, A.E. (2016). Ecological perspectives of hydrogen fermentation by microbial consortia: What we have learned and the way forward. International journal of hydrogen energy. 41, 17297 -17308.

Pakarinen, O., Lehtomäki, A., \& Rintala, J. (2008). Batch dark fermentative hydrogen production from grass silage: the effect of inoculum, $\mathrm{pH}$, temperature and $\mathrm{VS}$ ration. Int $J$ Hydrogen Energy; 33, 594-601.

Pandey A., Chang J., Hallenbeck P., \& Larroche C. (2013). Biohydrogen. 1st ed. Amsterdam, Netherlands: Elsevier.

Pérez-Rangel, M., Quiroz-Figueroa, F. R., González-Castañeda, J., \& Valdez-Vazquez, I. (2015). Microscopic analysis of wheat straw cell wall degradation by microbial consortia for hydrogen production. International Journal of Hydrogen Energy, 40(1), 151-160.
Quéméneur, M., Bittel, M., Trably, E., Duma,s C., Fourage L., Ravot G., Steyer J. P., \& Carrérea H. (2012). Effect of enzyme addition on fermentative hydrogenproduction from wheat straw. International journal of hydrogen energy, 3710639-10647.

SAGARPA, (2017). Planeación Agrícola Nacional 2017-2030, Jitomate mexicano. $1^{\text {a }}$ Edición, México.

Shade, A., Peter, H., Allison, S.D., Baho, D.L., Berga, M., Bürgmann, H., Huber, D.H., Langenheder, S., Lennon, J.T., Martiny, J.B., Matulich, K.L., Schmidt, T.M., \& Handelsman, J. (2012). Fundamentals of microbial community resistance and resilience. Front Microbiol 3, 1-19.

Tracy, B.P., Jones, S.W., Fast, A.G., Indurthi, D.C., \& Papoutsakis, E.T. (2012) Clostridia: the importance of their exceptional substrate and metabolite diversity for biofuel and biorefinery applications. Curr Opin Biotechnol 23, 364-381.

Ueno, Y., Haruta, S., Ishii, M., \& Igarashi, Y. (2001). Characterization of a microorganism isolated from the effluent of hydrogen fermentation by microflora. Journal of Bioscience and Bioengineering, 92, 397-400.

van Soest, P.J., Robertson J.B., \& Lewis B.A. (1991). Methods for Dietary Fiber, Neutral Detergent Fiber, and Nonstarch Plysaccharides in Relation to Animal Nutrition. Journal of Dairy Sciences, 74, 3583-3597.

Vanwonterghem, I., Jensen, P.D., Dennis, P.G., Hugenholtz, P. Rabaey, K., \& Tyson, G.W. (2014a). Deterministic processes guide long term synchronised population dynamics in replicate anaerobic digesters. ISME J, 8, 1-14.

Vanwonterghem, I., Jensen, P.D., Ho, D.P., Batstone, D.J., \& Tyson G.W. (2014b). Linking microbial community structure, interactions and function in anaerobic digesters using new molecular techniques. Curr Opin Biotechnol 27, 55-64.

Wang, C., Lin, P., \& Chang, J. (2006). Fermentative conversión of sucrose and pineapple waste into hydrogen gas in phosphatebuffered culture seeded with municipal sewage sludge. Process Biochemestry, 41(6): 13531358 .

CAMARENA-MARTÍNEZ, Sarai, MARTÍNEZ-MARTÍNEZ, Juan Humberto, SALDAÑA-ROBLES, Adriana y RUIZ-AGUILAR, Graciela M.L. Producción de Hidrógeno mediante digestión anaerobia de residuos de planta de jitomate. Revista de Energía Química y Física. 2019. 
Wang, J., \& Wan, W., (2009). Factors Influencing Fermentative Hydrogen Production: A Review, International Journal of Hydrogen Energy, 34, 799-811.

Xu, J.-F., Mi, Y.-T., \& Ren, N.-Q. (2016). Buffering action of acetate on hydrogen production by Ethanoligenens harbinense B49. Electronic Journal of Biotechnology, 23, 7-11

Zhang, M.L., Fan, Y.T., Xing, Y., Pan, C.M., Zhang, G.S., \& Lay, J.J. (2007). Enhanced biohydrogen production from cornstalk wastes with acidification pretreatment by mixed anaerobic cultures. Biomass and Bioenergy, 31(4), 250-254.

Zhang, L., Q. Ban, J. Li, Y. Xu, (2014). Assessment of effects of yeast extract on biohydrogen production from anaerobic activated sludge. Int. J. Agric. Biol., 16: 1189-1193.

Zhao, T., Xu, J. L., Chen, H., \& Li, Y. F. (2010). Effects of Phosphate Buffer Solution on Fermentative Biohydrogen Production of Biohydrogenbacterium R3 sp.nov. Advanced Materials Research, 113-116, 2185-2188. 